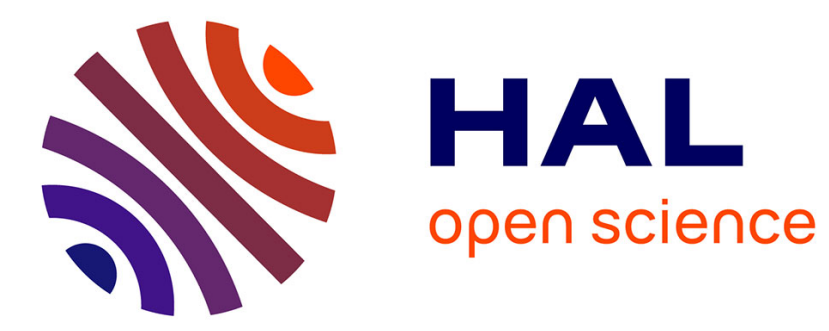

\title{
Topological Structures in Computer-Aided Music Analysis
}

Louis Bigo, Moreno Andreatta

\section{To cite this version:}

Louis Bigo, Moreno Andreatta. Topological Structures in Computer-Aided Music Analysis. David Meredith. Computational Music Analysis, Springer, pp.57-80, 2015, 978-3-319-25931-4. 10.1007/9783-319-25931-4_3 . hal-01263349

\section{HAL Id: hal-01263349 https://hal.science/hal-01263349}

Submitted on 30 Nov 2020

HAL is a multi-disciplinary open access archive for the deposit and dissemination of scientific research documents, whether they are published or not. The documents may come from teaching and research institutions in France or abroad, or from public or private research centers.
L'archive ouverte pluridisciplinaire HAL, est destinée au dépôt et à la diffusion de documents scientifiques de niveau recherche, publiés ou non, émanant des établissements d'enseignement et de recherche français ou étrangers, des laboratoires publics ou privés. 


\title{
Chapter 3 \\ Topological Structures in Computer-Aided Music Analysis
}

\author{
Louis Bigo and Moreno Andreatta
}

\begin{abstract}
We propose a spatial approach to musical analysis based on the notion of a chord complex. A chord complex is a labelled simplicial complex which represents a set of chords. The dimension of the elements of the complex and their neighbourhood relationships highlight the size of the chords and their intersections. Following a well-established tradition in set-theoretical and neo-Riemannian music analysis, we present the family of $T$ / I complexes which represent classes of chords which are transpositionally and inversionally equivalent and which relate to the notion of Generalized Tonnetze. A musical piece is represented by a trajectory within a given chord complex. We propose a method to compute the compactness of a trajectory in any chord complex. Calculating the trajectory compactness of a piece in $T / I$ complexes provides valuable information for music analysis and classification. We introduce different geometrical transformations on trajectories that correspond to different musical transformations. Finally, we present HexaChord, a software package dedicated to computer-aided music analysis with chord complexes, which implements most of the concepts discussed in this chapter.
\end{abstract}

Louis Bigo

Department of Computer Science and Artificial Intelligence, University of the Basque Country UPV/EHU, San Sebastián, Spain

e-mail: louis.bigo@ehu.eus

Moreno Andreatta

CNRS, France

IRCAM, Paris, France

Université Pierre et Marie Curie, Paris, France

e-mail: moreno.andreatta@ircam.fr 

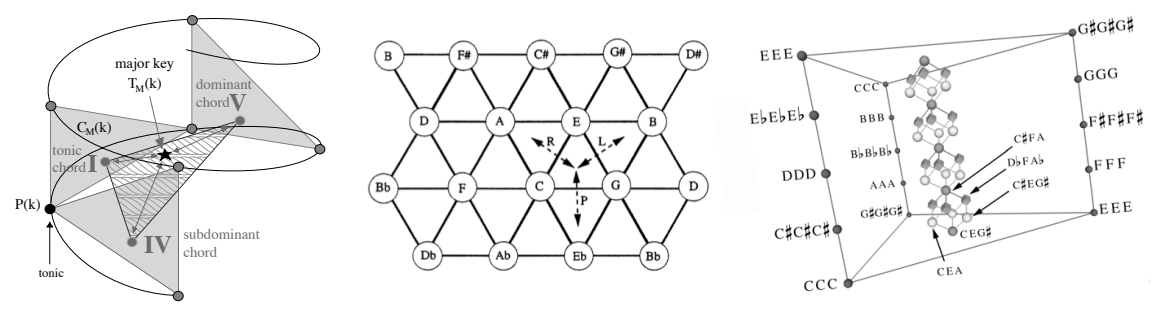

Fig. 3.1 Three symbolic spaces dedicated to musical representations: the spiral array (left), the Tonnetz (centre) and a voice leading space (right)

\subsection{Introduction}

This work concentrates on the harmonic aspect of musical sequences and introduces a spatial approach for its analysis. Musical analysis often requires specific tools when it focuses on a particular musical layer. For example, some theorists represent musical objects and their harmonic properties by (or in) symbolic spaces. When used in an analytic context, these spatial representations can reveal some strategies used to compose a piece. The spiral array (Chew, 2002), the Tonnetz (Cohn, 1997) and voice-leading spaces (Callender et al., 2008) are examples of such spaces. Among their numerous properties, they are well adapted for determining key boundaries, representing neo-Riemannian operations and voice-leading motions, respectively. Such spaces typically represent pitches or chords by vertices in a graph.

The chord spaces presented in this study include elements of higher dimension than vertices and edges. We represent $n$-note chords by geometrical objects of dimension $(n-1)$, called simplices. The faces of a simplex represent all sub-chords contained in the chord. Chord sets are represented by simplicial complexes. The possibly high dimension of a complex highlights specific neighbourhood relationships between chords and allows the space to represent more advanced musical properties.

A strong motivation of this work is the general desire to represent a collection of musical objects by a symbolic space governed by a set of neighbourhood relationships that reflect how represented objects interact. This general idea is inspired by the MGS project (Giavitto and Michel, 2001), which aims to provide tools for the modelling and simulation of (not necessarily musical) dynamical systems exhibiting dynamical structures.

Section 3.2 begins by briefly presenting the historical background of music representations, including a discussion of Pousseur's pioneering attempt to capture musical logic with geometric representations. We then present the notions of T/I class and Generalized Tonnetze. Section 3.3 introduces the notion of simplicial complex and explains how this concept allows for any set of chords to be represented by a symbolic space called a chord complex. This section finally introduces $T / I$ complexes which are chord complexes representing chords of a T/I class. Section 3.4 introduces the idea of representing musical sequences as trajectories in chord complexes. We introduce 
the notion of the $d$-compactness of such a trajectory as a heuristic for selecting chord complexes for analysis or classification. We finally present a method to transform a musical sequence by applying some geometric transformations on its trajectory. Chord complexes and trajectories have been implemented in the HexaChord music analysis software, which is presented and discussed in Sect. 3.5.

\subsection{Background in Musical Representations}

We present two well-known notions in music theory: the Tonnetz and T/I classes. The first is a spatial organization of pitches that constitutes a well-established analytical tool in the so-called neo-Riemannian music-theoretical tradition (Cohn, 2012; Tymoczko, 2012). The second provides a classification of musical chords based on their equivalence up to transposition and inversion. These two representation tools constitute the musical starting point of this work.

\subsubsection{The Tonnetz}

One of the strongest motivations of this work is the wish to formalize a widely used tool in music theory, analysis and composition: the Tonnetz. The Tonnetz is a symbolic organization of pitches in the Euclidean space defined by infinite axes associated with particular musical intervals. It was first investigated by Euler (1739) for acoustical as well as graph-theoretical purposes ${ }^{1}$ and rediscovered later by the musicologists A. von Oettingen and H. Riemann and by the composer and musictheorist H. Pousseur. More recently, music theorists have shown a strong interest in this model, in particular to represent typical post-romantic chord progressions (Cohn, 2012) currently called neo-Riemannian transformations. This model has also been used in musical composition, not only in contemporary musical styles (Chouvel, 2009) but also in more popular styles (Bigo and Andreatta, 2014).

The neo-Riemannian Tonnetz (on the left in Fig. 3.2) is a graph in which pitches are organized along the intervals of the perfect fifth (horizontal axis), major and minor thirds (diagonal axes). This representation has the interesting property that major and minor triads appear as triangles. Many theorists have investigated different derivations of the Tonnetz, often referred to as generalized Tonnetze. For instance, the three-dimensional Tonnetz introduced by Gollin (1998) is shown on the right in Fig. 3.2. This model corresponds to the one on the left side of Fig. 3.2 with some

\footnotetext{
${ }^{1}$ Euler's Tonnetz (Speculum Musicum) organizes pitches in just intonation along horizontal and vertical axes associated with the pure fifth and pure major third, respectively. Interestingly, the kind of music-theoretical problems he suggested could be approached via his Speculum Musicum deeply resonate with purely mathematical problems, such as the Königsberg Bridge Problem. This fact supports the idea that not only does mathematics apply to music but, conversely, music often anticipates some developments of mathematics (Andreatta, 2008).
} 


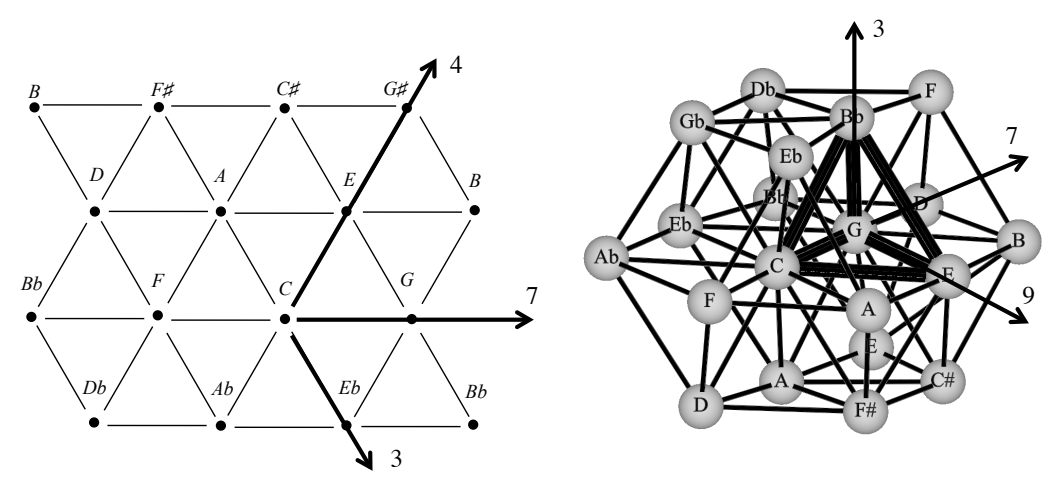

Fig. 3.2 On the left, a region of the neo-Riemannian Tonnetz. On the right, a three-dimensional derivation of the Tonnetz by Gollin (1998)

additional interval axes. Tetrahedra represent dominant seventh and half-diminished chords. Three-dimensional models are well adapted to the study of 4-note chord progressions. Similar structures can be built by associating axes with intervals that are diatonic instead of chromatic. In these diatonic Tonnetze, investigated by Cohn (2012), vertices and shapes only represent notes and chords belonging to a unique diatonic scale.

In this work, we limit ourselves to the context of equal temperament and octave equivalence, i.e., we are dealing with pitch classes. For example, the pitches $C \sharp 3, C \sharp 4$ and $\mathrm{D} b 4$ are all members of the same pitch class. In particular, what we call Tonnetz more exactly refers to the pitch-class Tonnetz. In this context, the graph on the left of Fig. 3.2 repeats infinitely the 12 pitch classes along its axes. The pitch-class Tonnetz is frequently represented as a toroidal structure by merging the nodes representing the same pitch classes (Cohn, 1997).

Interestingly, this geometrical structure has also been rediscovered independently from the neo-Riemannian music-analytical tradition by the composer and music theorist H. Pousseur, one of the leading figures, with P. Boulez and K. Stockhausen, of European integral Serialism. Although Pousseur's model is much less well-known and rarely cited in neo-Riemannian music analysis, it contains the roots of the spatial approach that we present in this chapter. The point of departure of Pousseur's network theory is Rameau's (1722) Traité de l'harmonie, in which the latter introduces a bidimensional representation of the tone-space in just intonation as generated by the pure fifths (horizontal axis) and pure major thirds (descending vertical axis). This representation is equivalent to the Speculum Musicum which was introduced by Euler a few years later. Pousseur first presented his Rameau-inspired geometrical model in 1968 (Pousseur, 1968), but he provides several analytical examples of what he calls his "Network technique" in a more recent paper (Pousseur, 1998). In this analytical essay he goes back to the definition of a Network as a spatial distribution of pitches (and pitch classes) according to at least two axes which are characterized as a chain 


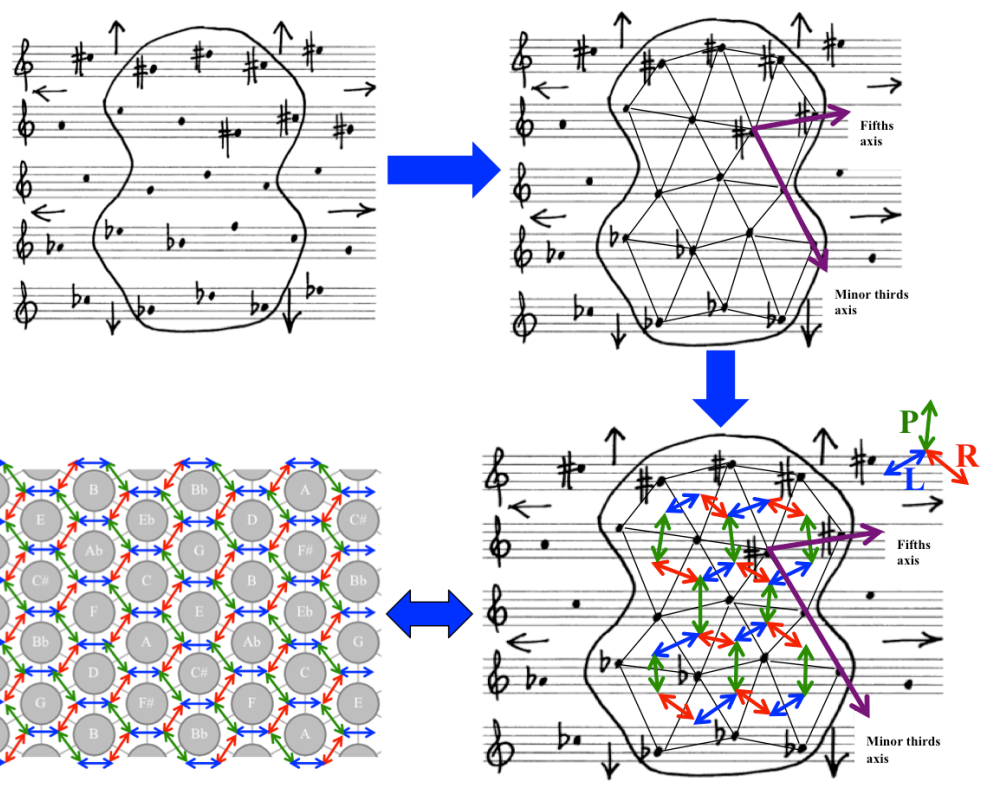

Fig. 3.3 The pitch network introduced by H. Pousseur in the late 1960s and accompanied by a triangulation showing its equivalence to the traditional Tonnetz.

of a single interval. It is easy to show that his pitch distribution is equivalent to the traditional Tonnetz structure as generated by the fifths and minor thirds axes, as shown in Fig. 3.3. As clearly pointed out by Pousseur, this geometrical representation is one of the possible network configurations which can be utilized in music analysis. The basic principle of the Network technique, as he claims, resides in choosing the generating axes, and hence constructing the triangulations of the space, in such a way that the musical relations between adjacent pitches (both harmonic and melodic) produce the most compact configuration within the given space. This clearly suggests that his Network technique is deeply rooted in a spatial approach to music analysis, which we can properly formalize in terms of simplicial complexes as presented in Sect. 3.3. Moreover, as Pousseur suggests, the music analyst can transform the underlying geometrical space and move from a given network to a different one by simply changing the generating axes, which modifies the hierarchy of intervallic relations as well as the structural proximity between the pitches (or pitch classes). The next two sections will show how to transform this original compositional and music-theoretical intuition into a computational analytical model. 


\subsubsection{Generalized Tonnetze and T/I Classes}

The highlighting of particular chords (minor/major chords as triangles in the neoRiemannian Tonnetz and dominant seventh/half diminished chords as tetrahedra in the three-dimensional Tonnetz) suggests the idea that the starting point of the construction of a Tonnetz could be a set of chords rather than a set of interval axes. In the two examples above, the represented chords in a Tonnetz are all transpositionally and inversionally equivalent - that is, they belong to the same T/I class (Forte, 1973). This property comes from the repetition and the invertibility of the intervals on the axes.

It is common to identify a T/I class by the intervallic structure which is shared by all the chords of the class. For instance, the 24 major and minor chords all share the intervallic structure $[3,4,5]$ because the row of intervals between the pitch classes they are resulting from is composed of a minor third ( 3 semitones), a major third (4 semitones) and a fourth ( 5 semitones). This notation of the intervallic structure is defined up to reflection and circular permutation. Intervals are ordered in the opposite direction (up to a cyclic permutation) for major $([4,3,5])$ and minor chords $([3,4,5])$. Intervallic structures can be diatonic as well. In this case, the intervals add up to 7 instead of 12. For example, the triads on the seven degrees of the diatonic scale belong to the class identified by the intervallic structure [2,2,3]. Even though T/I classes can be enumerated for any division of the octave (i.e., for any $N$ ), we focus in this work on the diatonic $(N=7)$ and chromatic $(N=12)$ systems. $^{2}$

There exist 224 such classes in the chromatic system, also known as Forte classes (Forte, 1973). In the diatonic system, which divides the octave into seven (non-equal) parts, there exist 18 such classes. Following this line, we are interested in building the generalized Tonnetze associated with the $224 \mathrm{~T} / \mathrm{I}$ chromatic classes and $18 \mathrm{~T} / \mathrm{I}$ diatonic classes.

\subsection{Generalized Tonnetze as Chord Complexes}

This section presents a method to represent any arbitrary set of chords by a multidimensional structure called a chord complex. Figure 3.7 illustrates an example of a chord complex representing a set of four chords. A chord complex is a simplicial complex whose components, called simplices, are labelled by chords. ${ }^{3}$ In a chord complex, the dimension of the simplices represents the size of the chords. Furthermore, the intersections of the simplices represent the common pitch classes of the chords. Chord complexes representing the chords of a T/I class are called T/I complexes and relate to Generalized Tonnetze.

\footnotetext{
${ }^{2}$ For enumeration and classification purpose, T/I classes can be associated with the orbits of the action of the dihedral group $\mathbb{D}_{N}$ on the subsets of $\mathbb{Z}_{N}$, as described by Andreatta and Agon (2003).

${ }^{3}$ Simplicial complexes belong to a more general family of spaces called cellular complexes, which have already proved to be useful in music theory (Bigo et al., 2011).
} 

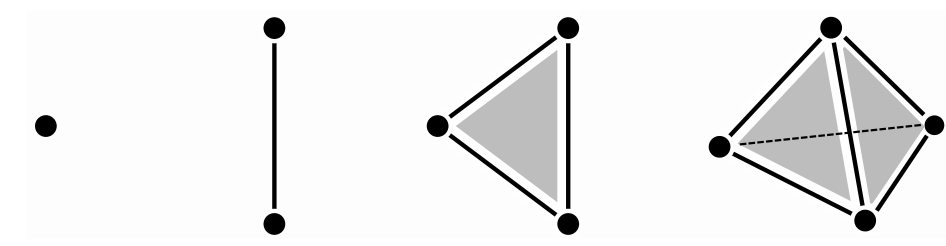

Fig. 3.4 From left to right: a 0-simplex, a 1-simplex, a 2-simplex and a 3-simplex represented with their closure

\subsubsection{Simplicial Complexes}

A simplicial complex is a multidimensional space built by gluing together more elementary spaces called simplices. A simplex (more precisely a $d$-simplex) is the abstraction of a space of dimension $d$. As illustrated in Fig. 3.4, a 0-simplex corresponds to a vertex, a 1-simplex corresponds to an edge, a 2-simplex to a triangle, a 3 -simplex to a tetrahedron, etc. In a simplicial complex, a $d$-simplex is necessarily incident to $d+1$ simplices of dimension $d-1$. For instance, a 1-simplex (i.e., an edge) is incident to two 0 -simplices (i.e., 2 vertices), a 2 -simplex (i.e., a triangle) is incident to three 1-simplices (i.e., 3 edges), etc. This recursive property defines the closure of a simplex, consisting of all its incident simplices of lower dimension. In a simplicial complex, a subset of simplices that itself constitutes a simplicial complex is called a sub-complex.

A simplicial d-complex is a simplicial complex where the highest dimension of any simplex is $d$. A graph is a simplicial 1-complex. For any natural number $n$, the $n$-skeleton of a simplicial complex $\mathcal{K}$ is defined by the sub-complex $\mathcal{S}_{n}(\mathcal{K})$ of this complex formed by its simplices of dimension $n$ or less. Figure 3.5 illustrates a simplicial 3-complex and its 1-skeleton.

The $f$-vector of a simplicial $d$-complex corresponds to the finite sequence $\left(f_{1}, \ldots, f_{d+1}\right)$ where $f_{p+1}$ is the number of $p$-simplices included in the complex. The $f$-vector of the complex in Fig. 3.5 is $(11,17,7,1)$ because it includes 11 ver-
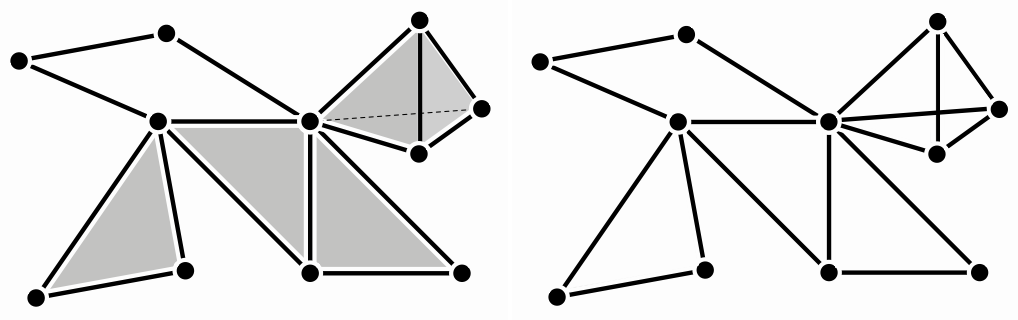

Fig. 3.5 A simplicial 3-complex (on the left) and its 1-skeleton (on the right) 

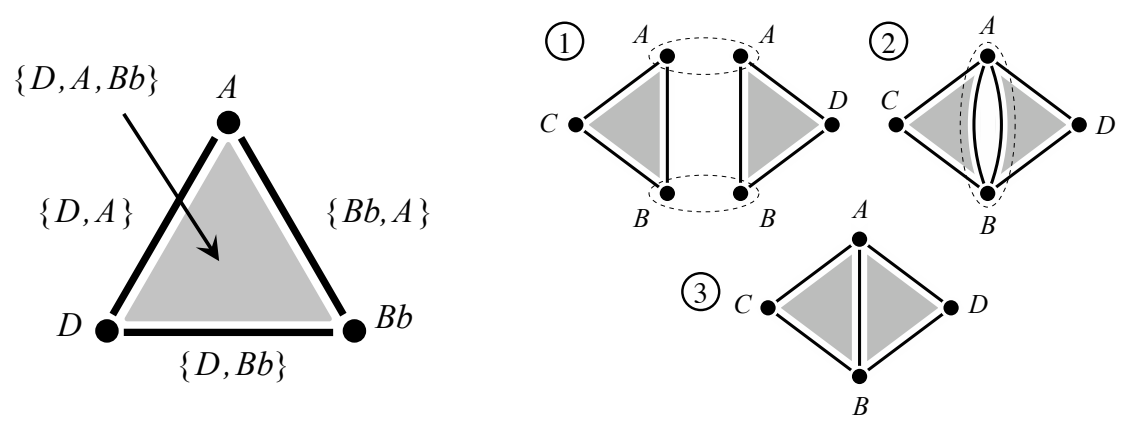

Fig. 3.6 On the left, the 2-simplex $\mathcal{S}(C)$ representing the chord $C=\{D, A, B b\}$ and all sub-chords and notes included in it. On the right, the identification of boundaries illustrating the self-assembly process on the 2 -simplices representing the chords $\{C, A, B\}$ and $\{D, A, B\}$

tices, 17 edges, 7 triangles and one tetrahedron. The term $f_{p}$ of the $f$-vector of a complex $\mathcal{K}$ is denoted by $f_{p}(\mathcal{K})$.

Simplicial collections are simplicial complexes in which every simplex is labelled by an arbitrary value. The left side of Fig. 3.6 illustrates a simplicial collection of dimension 2. A simplicial collection can be built from a set of labelled simplices by applying a self-assembly process (Giavitto and Spicher, 2008). This process is based on the identification of the simplex boundaries that share the same labels. This topological operation holds in all dimensions. The right side of Fig. 3.6 illustrates the process on two 2 -simplices. In step 1, nodes $A$ and $B$ are merged. Then, in step 2 , the resulting edges $\{A, B\}$ are merged. The final structure is a connected, twodimensional, simplicial collection in which every simplex is labelled with its own value (step 3).

\subsubsection{Chord Complexes}

We use a method described by Bigo et al. (2011) to represent a set of chords by a simplicial collection that we call a chord complex. In this representation, chords are reduced to pitch class sets. This requires some abstraction since some properties of chords as they actually occur within a musical context (e.g., the octave and duration of each note) are not represented. An $n$-pitch class set (i.e., a set of $n$ pitch classes) is represented by an $(n-1)$-simplex. In particular, a 0 -simplex represents a single pitch class, a 1-simplex a 2-pitch class set, etc. We denote by $\mathcal{S}(A)$ the simplex representing the pitch class set $A$. The simplices constituting the closure of $\mathcal{S}(A)$ represent all the sub-chords of $A$. The 2-simplex representing the pitch class set $\{D, A, B b\}$ is illustrated on the left of Fig. 3.6. A chord complex is built by:

1. representing each chord of the collection by a simplex as described above, 

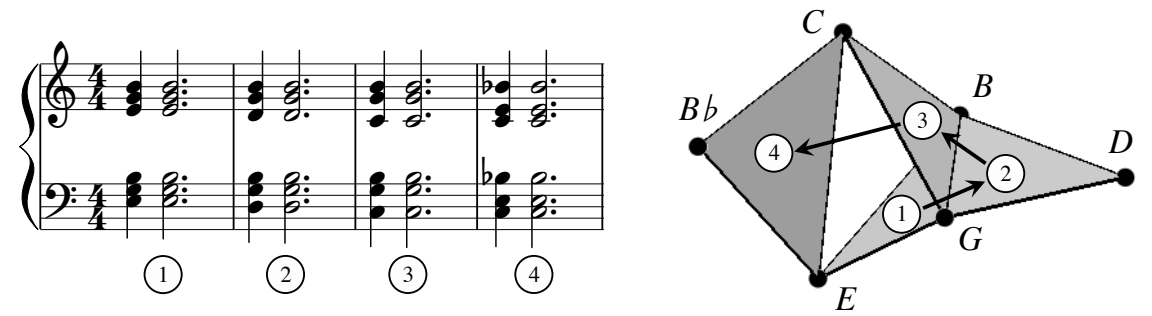

Fig. 3.7 First measures of Metamorphosis by Philip Glass (left) represented by a chord complex (right). The three arrows provide the order in which the four chords are played

2. applying the self-assembly process to the resulting collection of simplices.

This method ensures that a given chord cannot be represented more than once in the simplicial complex. As an example, on the right in Fig. 3.7 is shown the complex resulting from the assembly of the four introductory chords of the piano piece Metamorphosis by Philip Glass, shown on the left in the same figure. The four chords are respectively associated with the pitch class sets $\{E, G, B\},\{D, G, B\},\{C, G, B\}$ and $\{C, E, B b\}$. Each chord includes three pitch classes and is represented by a 2simplex, that is, a triangle. The whole complex exhibits the intersections between the four pitch class sets.

This representation method provides a topological signature for any chord sequence. The musical interpretation of topological properties of chord complexes (dimension, size, connectedness, morphisms, etc.) is discussed by Bigo (2013). In the following, we will focus on chord complexes representing sets of chords related by some theoretic properties (for example chords belonging to the same T/I class) rather than sets of chords included in a given musical sequence as illustrated by the example in Fig. 3.7.

\subsubsection{T/I Complexes}

We represent a generalized Tonnetz as a chord complex composed of $n$-simplices representing the chords of a given T/I class. In the following, we consider in particular $\mathcal{K}_{T I}\left[a_{1}, \ldots, a_{i}\right]$, the complex associated with the T/I class identified by the intervallic structure $\left[a_{1}, \ldots, a_{i}\right]$. To build such a complex, we first enumerate all the chords belonging to the T/I class, we then build the corresponding complex, as explained in Sect. 3.3.2. As a consequence, the dimension of a T/I complex corresponds to the size of its intervallic structure minus one.

Figure 3.8 illustrates the diatonic complexes $\mathcal{K}_{T I}[2,5], \mathcal{K}_{T I}[2,2,3]$ and $\mathcal{K}_{T I}[1,2,2,2]$ associated with the scale of $C$ major. These complexes were described by Mazzola et al. (2002) as interpretations of the diatonic scale. They have the topology of a circle, a Möbius strip and a torus, respectively. An alternative represen- 


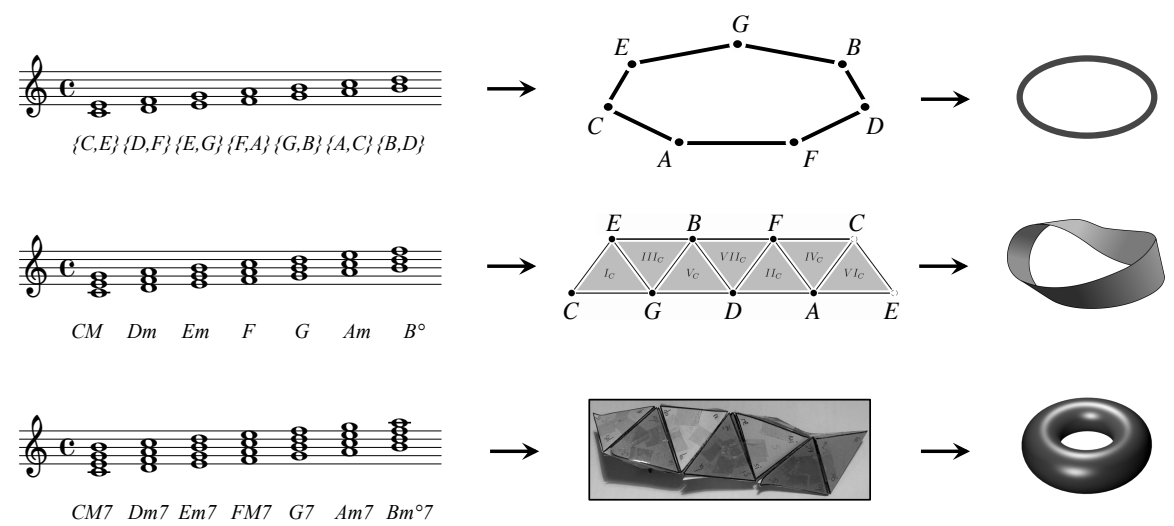

Fig. 3.8 Construction of the diatonic complexes $\mathcal{K}_{T I}[2,5], \mathcal{K}_{T I}[2,2,3]$ and $\mathcal{K}_{T I}[1,2,2,2]$ by assembling degrees of the tonality of $C$ major (Mazzola et al., 2002)

tation of the complex $\mathcal{K}_{T I}[1,2,4]$ in which all pitch classes of the diatonic scale are neighbours has been used for music analysis by Hook (2014).

Figure 3.9 illustrates the chromatic complexes $\mathcal{K}_{T I}[2,5,5]$ and $\mathcal{K}_{T I}[3,4,5]$ which result from the assembly of suspended chords (Sus4 chords) and major/minor chords respectively. The organization of pitch classes in $\mathcal{K}_{T I}[2,5,5]$ corresponds to the wellknown Wicki-Hayden note layout, which is used for the key layout on some keyboard instruments, such as the bandoneon. The 1-skeleton of $\mathcal{K}_{T I}[3,4,5]$ corresponds to the Tonnetz. Topological properties of T/I chord complexes of dimension 2 were studied by Catanzaro (2011). Although the present study focuses on T/I complexes (i.e., $\left.\mathcal{K}_{T I}[\cdot]\right)$ due to their relation with Generalized Tonnetze, this approach can be generalized to enumerate chord complexes defined by any equivalence relation, not
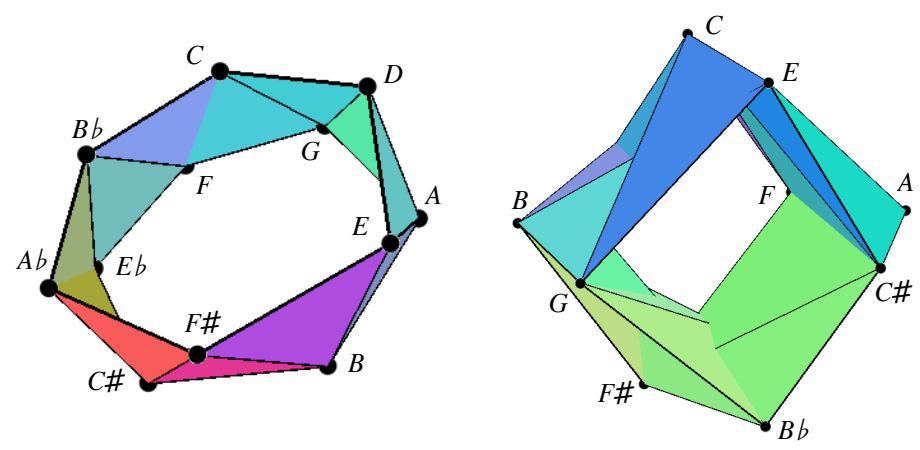

Fig. 3.9 On the left, the complex of the suspended chords $\left(\mathcal{K}_{T I}[2,5,5]\right)$ of size 12 . On the right, the complex of major and minor chords $\left(\mathcal{K}_{T I}[3,4,5]\right)$ of size 24 , whose 1 -skeleton corresponds to the Tonnetz. The first complex is a strip and the second is a torus 
just transposition and inversion. For example, complexes of chords equivalent up to transposition only $\left(\mathcal{K}_{T}[\cdot]\right)$, up to transposition and interval permutation $\left(\mathcal{K}_{T P}[\cdot]\right)$ and up to affine transformation $\left(\mathcal{K}_{M}[\cdot]\right)$ have been enumerated and classified in a catalogue (Bigo, 2013). ${ }^{4}$

\subsubsection{Unfolded Representations of T/I Complexes}

The neo-Riemannian Tonnetz was originally designed as a triangular graph embedded within a two-dimensional Euclidean space, as illustrated on the left in Fig. 3.2. One may imagine the triangulation as being generated by the choice of three axes representing three privileged intervallic directions starting from a referential initial point. In the case of Fig. 3.2 (left), we start from the note $\mathrm{C}$ and we consider three axes corresponding to the minor third, perfect fifth and major third intervals. Note that two axes suffice to generate the triangulation of the plane, since the interval corresponding to one of the axes is simply the sum of the intervals corresponding to the two remaining axes. In our case, for example, the horizontal axis, going from left to right, represents a fifth interval which is the sum of a minor third and a major third, corresponding, respectively, to the two diagonal directions within the graph. This property is easily generalized to the case of the three-dimensional Tonnetz, as represented on the right side of Fig. 3.2. In this case, three axes are necessary to generate the three-dimensional model and they correspond to the minor third, major sixth and perfect fifth intervals. Note that, in this case, the reference point is G, as indicated on the right side of Fig. 3.2. In both representations, pitch classes are replicated infinitely along the axes by applying successive transpositions of the same interval.

These spaces can equivalently be thought of as the result of two T/I chord complexes which have been unfolded in a Euclidean space. Starting from a given $n$-note chord considered as belonging to a T/I class, one can first represent it by an $(n-1)$-simplex. The simplex is then embedded in an equilateral manner in the $(n-1)$-dimensional Euclidean space. For a 3-note chord, this space is the Euclidean plane and the chord is embedded as an equilateral triangle. The directions given to the 1-simplices (i.e., edges) define axes associated with particular intervals, as in the Tonnetz. Then, simplices are naturally replicated along these axes, in such a way that the represented chords respect the transpositions induced by the intervals of the axes. By starting with a major chord and a dominant seventh chord, one obtains the complexes $\mathcal{K}_{T I}[3,4,5]$ and $\mathcal{K}_{T I}[2,3,3,4]$ which are then unfolded as shown in Fig. 3.10. A consequence of this generic method of construction is that two complexes associated with chord classes of the same size are represented by identical structures (they are said to be isomorphic). Figure 3.10 shows the unfolding of the

\footnotetext{
4 This "paradigmatic approach", where groups act as "paradigms" in the enumeration and classification of musical structures, corresponds to well-known catalogues of chords (respectively, the Vieru-Zalewsky catalogue of transpositional chord classes, Forte's pitch class sets catalogue, Estrada's permutohedron and Morris-Mazzola's affine orbits catalogue).
} 

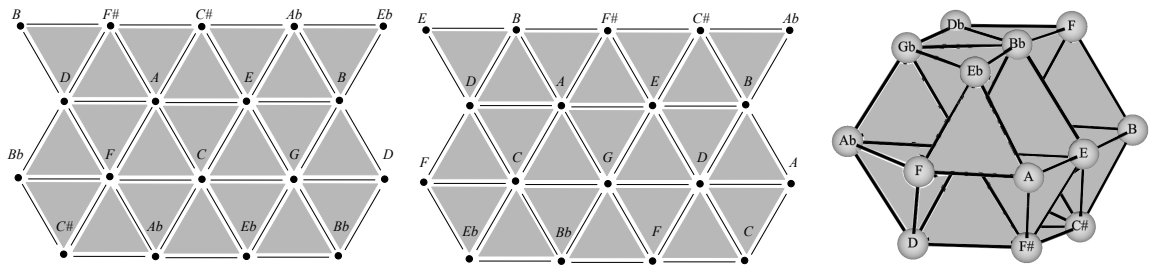

Fig. 3.10 Unfolded representations of 2-dimensional chord complexes $\mathcal{K}_{T I}[3,4,5]$ (left) and $\mathcal{K}_{T I}[2,3,7]$ (centre) as isomorphic structures. On the right, the unfolded representation of the 3 -dimensional chord complex $\mathcal{K}_{T I}[2,3,3,4]$ composed of dominant seventh and half-diminished chords

complexes $\mathcal{K}_{T I}[3,4,5]$ and $\mathcal{K}_{T I}[2,3,7]$, both corresponding to a two-dimensional infinite triangular tessellation.

\subsection{Analysis and Transformation of Trajectories in T/I Complexes}

In this section, we represent musical sequences by trajectories in T/I complexes. We first perform a harmony-based analysis and classification by studying the shape of these trajectories. We then present a variety of spatial transformations on these trajectories which result in musical transformations on the represented sequences.

\subsubsection{Representation of a Piece in a Chord Complex}

In this work, a trajectory corresponds to a temporal sequence of regions in a chord complex. Each successive region represents a temporal segment of the piece. We use a very simple segmentation method, based on appearance and disappearance of pitch classes. Each time a pitch class enters or leaves the set of played notes, the current segment stops and a new one begins. This principle is illustrated in Fig. 3.11. Note that a musical sequence could be represented by a trajectory constructed using any other segmentation process, including more sophisticated algorithms involving automatic harmonic analysis. Each segment is characterized by its relative duration, compared to the other segments. A musical sequence $P$ is thus reduced to a sequence of pitch class sets, each associated with a relative duration. We thus have $P=$ $\left[\left(A_{0}, d_{0}\right), \ldots,\left(A_{N}, d_{N}\right)\right]$ where $A_{i}$ is the set of active pitch classes during a duration $d_{i}$.

We represent the chord $A$ in the chord complex $\mathcal{K}$ by the sub-complex $\mathcal{K}_{A}$ of $\mathcal{K}$ consisting of all the simplices in $\mathcal{K}$ which are labelled by a pitch class set included in A. A trajectory in a chord complex $\mathcal{K}$ is a sequence of sub-complexes of $\mathcal{K}$, each of 


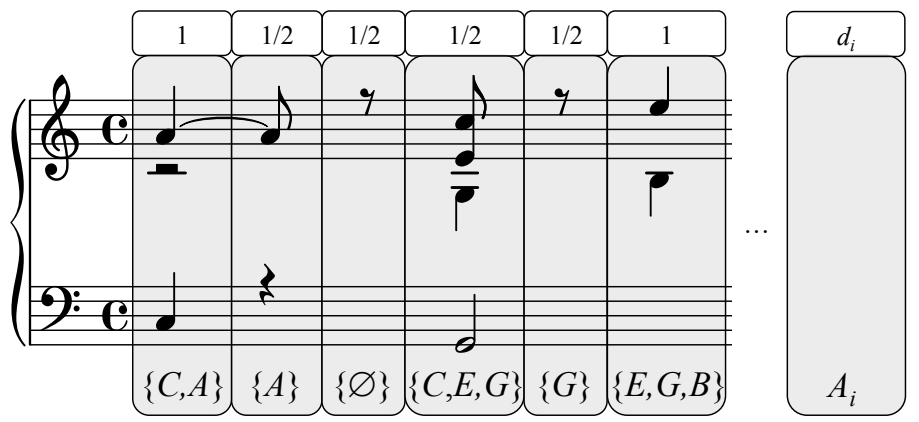

Fig. 3.11 Segmentation of a sequence depending on the set of played pitch classes. The duration of a segment (grey box) is calculated with respect to the quarter note taken as a unit

which is associated with a duration. The musical sequence $\left[\left(A_{0}, d_{0}\right), \ldots,\left(A_{N}, d_{N}\right)\right]$ is represented in a complex $\mathcal{K}$ by a trajectory $\left[\left(\mathcal{K}_{0}, d_{0}\right), \ldots,\left(\mathcal{K}_{N}, d_{N}\right)\right]$.

Figure 3.12 shows the sub-complexes constituting the trajectory of the sequence in Fig. 3.11 in $\mathcal{K}_{T I}[3,4,5]$. For more clarity, the trajectory is represented in the unfolded representation of the complex. Note that the 1-skeleton of this representation corresponds to the Tonnetz depicted on the left of Fig. 3.2.

Figure 3.13 shows the representation of the chorale, BWV 326, by J. S. Bach, by a trajectory in the $\mathrm{T} / \mathrm{I}$ complexes $\mathcal{K}_{T I}[3,4,5]$ and $\mathcal{K}_{T I}[1,4,7]$. The trajectory on the left constitutes a shape that is more compact than the trajectory on the right. This observation suggests the idea that the different $\mathrm{T} / \mathrm{I}$ complexes might not be equally

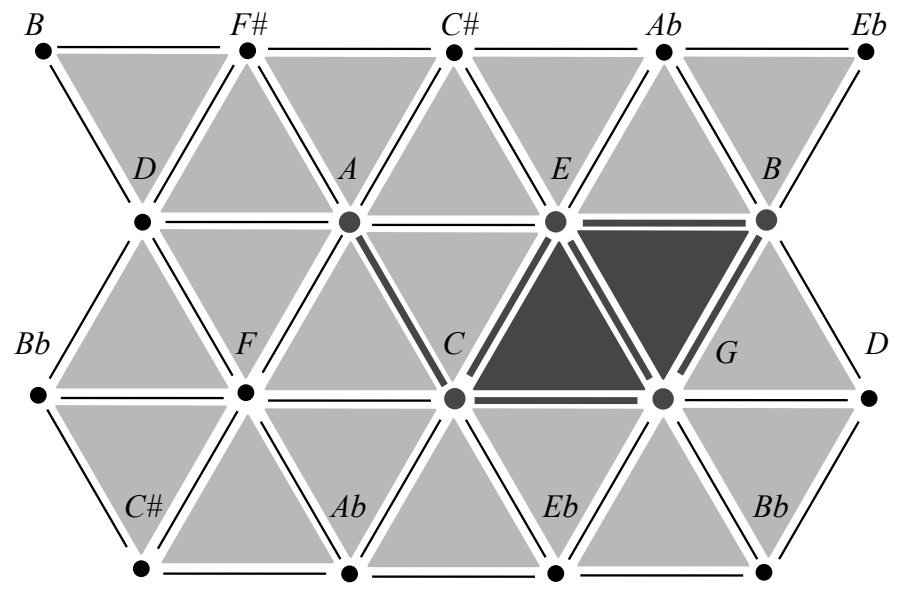

Fig. 3.12 Union (in dark grey) of the subcomplexes constituting a trajectory representing the sequence illustrated in Fig. 3.11 in $\mathcal{K}_{T I}[3,4,5]$. Note that the edge between $\mathrm{A}$ and $\mathrm{C}$ is also included in the union 

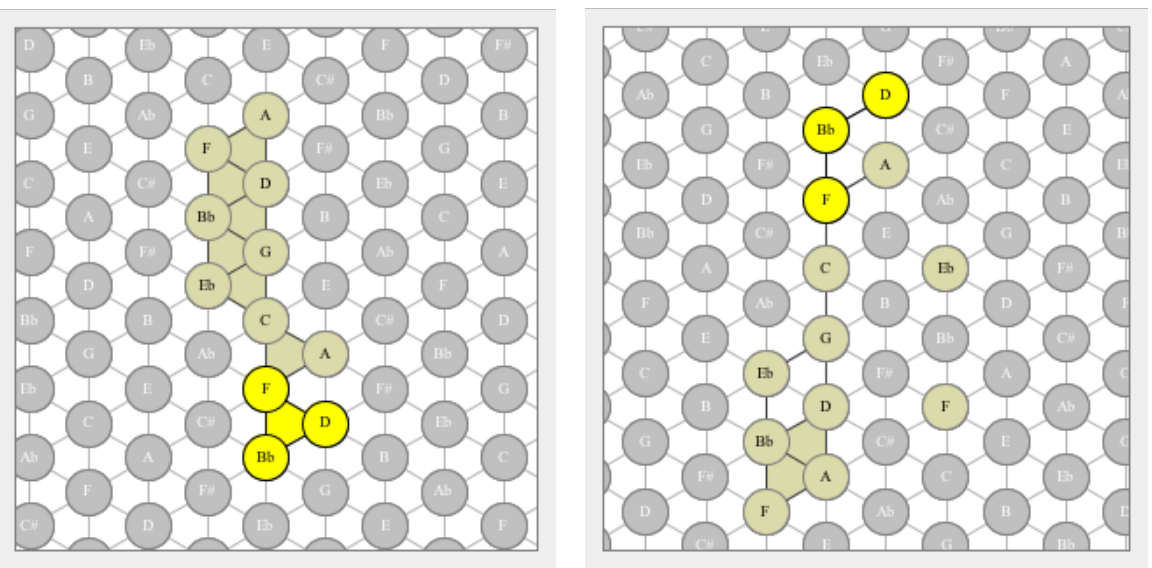

Fig. 3.13 First measures of the chorale, BWV 326, by J. S. Bach, represented as a trajectory in $\mathcal{K}_{T I}[3,4,5]$ (on the left) and in $\mathcal{K}_{T I}[1,4,7]$ (on the right)

well adapted to representing and analysing a particular musical sequence. In the next section, we propose a method for computing the compactness of a trajectory, in order to estimate how well a complex is adapted to representing a given musical sequence.

\subsubsection{Computing Trajectory Compactness}

In this section, we propose a method for computing the compactness of a trajectory representing a musical sequence in a chord complex.

\subsubsection{Chord Compactness}

Let $\mathcal{K}$ be a chord complex and $\mathcal{K}_{A}$ the sub-complex of $\mathcal{K}$ representing a pitch class set $A$. We define the compactness at the dimension $d$ (or the $d$-compactness) of $A$ in $\mathcal{K}$ to be

$$
\mathcal{C}_{d}(\mathcal{K}, A)=\frac{f_{d+1}\left(\mathcal{K}_{A}\right)}{f_{d+1}(\mathcal{S}(A))}
$$

In other words, the $d$-compactness corresponds to the number of $d$-simplices included in the representation of $A$ in $\mathcal{K}$ divided by the number of $d$-simplices included in the simplicial representation of $A .{ }^{5}$ Note that the value of the $d$-compactness varies between 0 and 1 whatever the dimension $d$ is. The value chosen for the parameter

\footnotetext{
5 This ratio recalls the global clustering coefficient (Holland and Leinhardt, 1971) in graph theory, which measures the degree to which nodes in a graph tend to cluster together. Our approach is more general because it applies to a set of arbitrary elements of the space (not necessarily neighbours of
} 

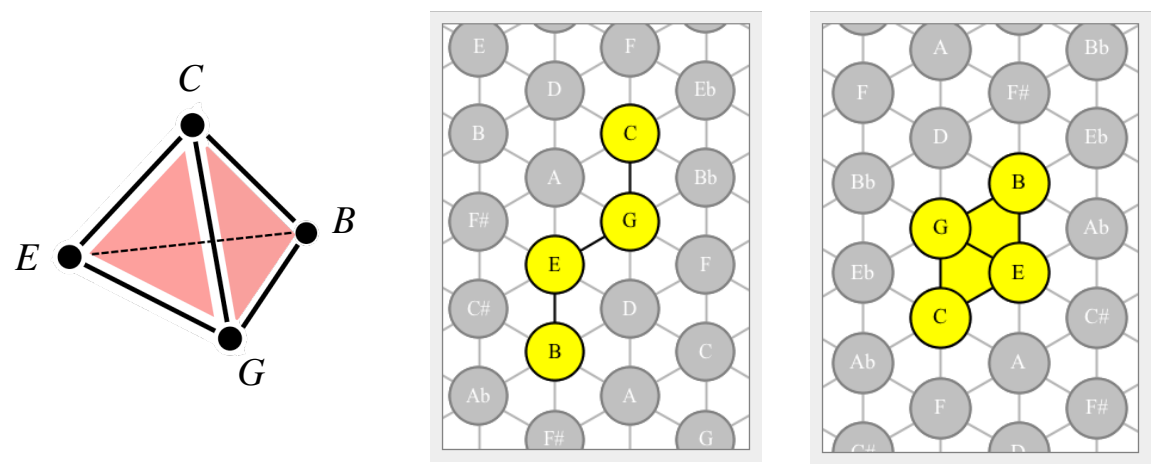

Fig. 3.14 The pitch class set $A=\{C, E, G, B\}$ represented by the 3-simplex $\mathcal{S}(A)$ (left), by a subcomplex of $\mathcal{K}_{T I}[2,3,7]$ (centre) and by a sub-complex of $\mathcal{K}_{T I}[3,4,5]$ (on the right)

$d$ in the computation of the compactness depends on the type of study which is being performed. 1-compactness will be calculated for a study based on intervals. If the study relates to the inclusion of chords of size 3 , one may prefer to work with 2-compactness.

As an example, let us compute the 1-compactness of the pitch class set $A=\{C, E, G, B\}$ in $\mathcal{K}_{T I}[2,3,7]$ and $\mathcal{K}_{T I}[3,4,5]$. As explained in Sect. 3.3.2, a 4pitch class set is represented by a 3 -simplex which includes six 1 -simplices (i.e., $\left.f_{2}(\mathcal{S}(A))=6\right)$. As Fig. 3.14 shows, the representation of $A$ in $\mathcal{K}_{T I}[2,3,7]$ includes three 1-simplices, whereas its representation in $\mathcal{K}_{T I}[3,4,5]$ includes five 1-simplices. We thus have $\mathcal{C}_{1}\left(\mathcal{K}_{T I}[2,3,7], A\right)=3 / 6=0.5$ and $\mathcal{C}_{1}\left(\mathcal{K}_{T I}[3,4,5], A\right)=5 / 6=0.83$. Analogously, by focusing on 2-simplices, we have $\mathcal{C}_{2}\left(\mathcal{K}_{T I}[2,3,7], A\right)=0 / 4=0$ and $\mathcal{C}_{2}\left(\mathcal{K}_{T I}[3,4,5], A\right)=2 / 4=0.5$. These results show that the representation of $A$ in $\mathcal{K}_{T I}[3,4,5]$ is more compact than in $\mathcal{K}_{T I}[2,3,7]$ (as can be seen in Fig. 3.14).

Figure 3.15 illustrates the 2-compactness of four types of chords commonly used in tonal music: major, major seventh, dominant seventh and diminished seventh. This diagram shows that the compactness of a chord can be more or less equally distributed among a set of different complexes. For example, the equal compactness of the dominant seventh chord in $\mathcal{K}_{T I}[2,3,7], \mathcal{K}_{T I}[2,4,6], \mathcal{K}_{T I}[3,3,6]$ and $\mathcal{K}_{T I}[3,4,5]$ shows the harmonic diversity of this chord. On the other hand, the diminished seventh chord is represented with strong compactness in $\mathcal{K}_{T I}[3,3,6]$ only. This is due to the fact that the 3-note sub-chords included in this chord all belong to the T/I class $[3,3,6]$.

This type of diagram provides an original description of the constitution of a chord and can be exploited to represent Z-relations between chords (Mandereau et al., 2011).

the same element) and in a simplicial complex of any dimension (graphs are simplicial complexes of dimension 1). 


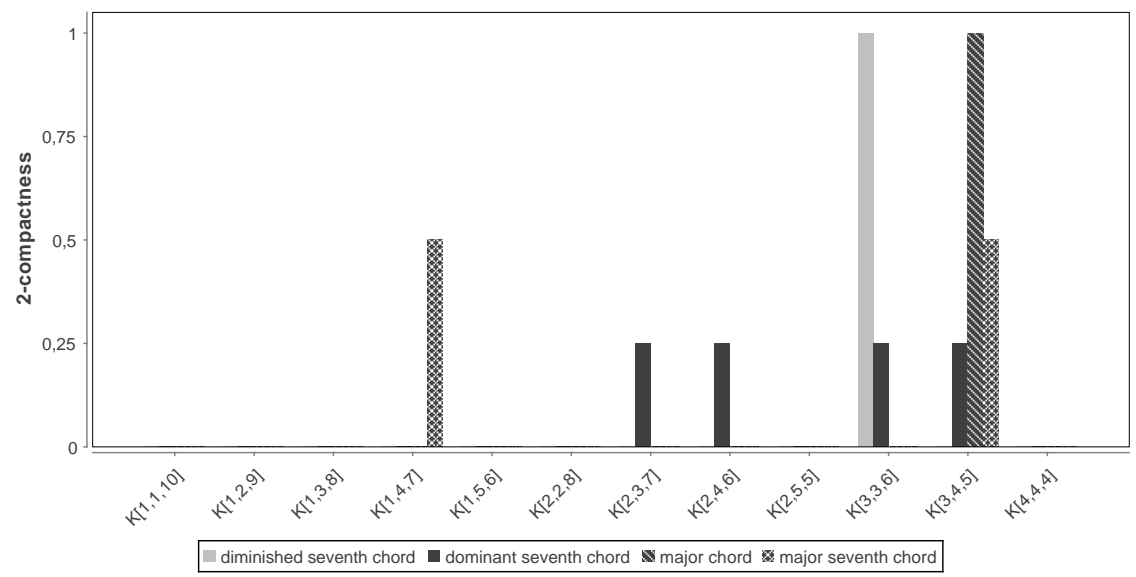

Fig. 3.15 2-compactness of diminished seventh, dominant seventh, major and major seventh chords in the twelve two-dimensional T/I complexes

Figure 3.16 illustrates the average 2-compactness of the set of all 3-pitch class sets. The compactness is computed in the twelve two-dimensional T/I complexes. The irregularity of the histogram shows that T/I complexes do not tend to represent arbitrary chords with the same compactness. This property depends on the size of the complex. For example, the probability of a random chord being represented compactly in $\mathcal{K}_{T I}[3,4,5]$ (which includes twenty-four 2-simplices) is six times higher than in $\mathcal{K}_{T I}[4,4,4]$ (which includes only four 2 -simplices). The average value $E\left(\mathcal{C}_{d}(\mathcal{K}, A)\right)$ of the $d$-compactness $\mathcal{C}_{d}$ of chords of size greater than or equal to $d$ in a complex $\mathcal{K}$ is equal to the number of $(d+1)$-simplices in $\mathcal{K}$ divided by the total number of chords of size $d+1$ :

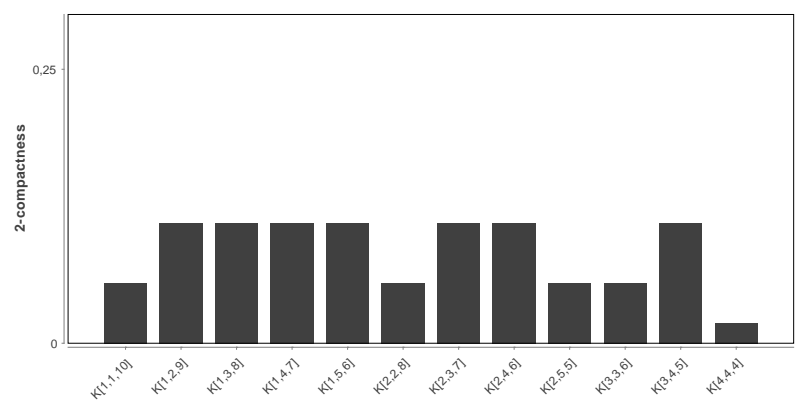

Fig. 3.16 Average 2-compactness of the set of all 3-pitch class sets in the twelve T/I complexes of dimension 2 


$$
E\left(\mathcal{C}_{d}(\mathcal{K}, A)\right)=\frac{f_{d+1}(\mathcal{K})}{\left(\begin{array}{c}
N \\
d+1
\end{array}\right)}
$$

\subsubsection{Trajectory Compactness}

The global $d$-compactness of a trajectory $T_{P}$ can be defined as the average of the $d$-compactness of its successive chords weighted by their duration $d_{i}$ :

$$
\mathcal{C}_{d}\left(\mathcal{K}, T_{P}\right)=\frac{1}{D} \times \sum_{i=0}^{N} d_{i} \mathfrak{C}_{d}\left(\mathcal{K}, \mathcal{K}_{i}\right),
$$

where $D=\sum_{i=0}^{N} d_{i}$ represents the duration of the whole piece.

In the following, the compactness of the trajectory of a piece in a complex will systematically be displayed in comparison with the average compactness of chords in that same complex.

\subsubsection{Computing the Compactness of Trajectories for Musical Analysis and Classification}

We now analyse a number of pieces in terms of the compactness of their trajectories in different $\mathrm{T} / \mathrm{I}$ complexes.

\subsubsection{Comparing Spaces Regarding a Piece}

The histograms in Fig. 3.17 illustrate the 2-compactness of the trajectories of three different pieces in the 12 chromatic T/I complexes of dimension 2. In each complex, the compactness of the trajectory of the piece (in black) is compared to the average compactness of its chords (in grey). The histograms have been generated with the software HexaChord which will be described in Sect. 3.5.

The first piece is the chorale, BWV 328, by J. S. Bach . The high compactness of the trajectory in $\mathcal{K}_{T I}[3,4,5]$ results from the strong use of major and minor chords which is typical of tonal music in general and Bach's chorales in particular. The high compactness of the trajectories in $\mathcal{K}_{T I}[2,3,7]$ and $\mathcal{K}_{T I}[2,5,5]$ is due to the use of dominant seventh and suspended chords which have particular functions in this style of music. The compactness in the complexes $\mathcal{K}_{T I}[2,2,8], \mathcal{K}_{T I}[2,4,6]$ and $\mathcal{K}_{T I}[4,4,4]$ of Claude Debussy's prelude, Voiles, highlights the predominant use of the whole-tone scale in this piece. Finally, the piece Parodie from Schoenberg's Pierrot Lunaire illustrates for each complex a compactness relatively close to the average compactness of chords. This results from an almost equally distributed use 

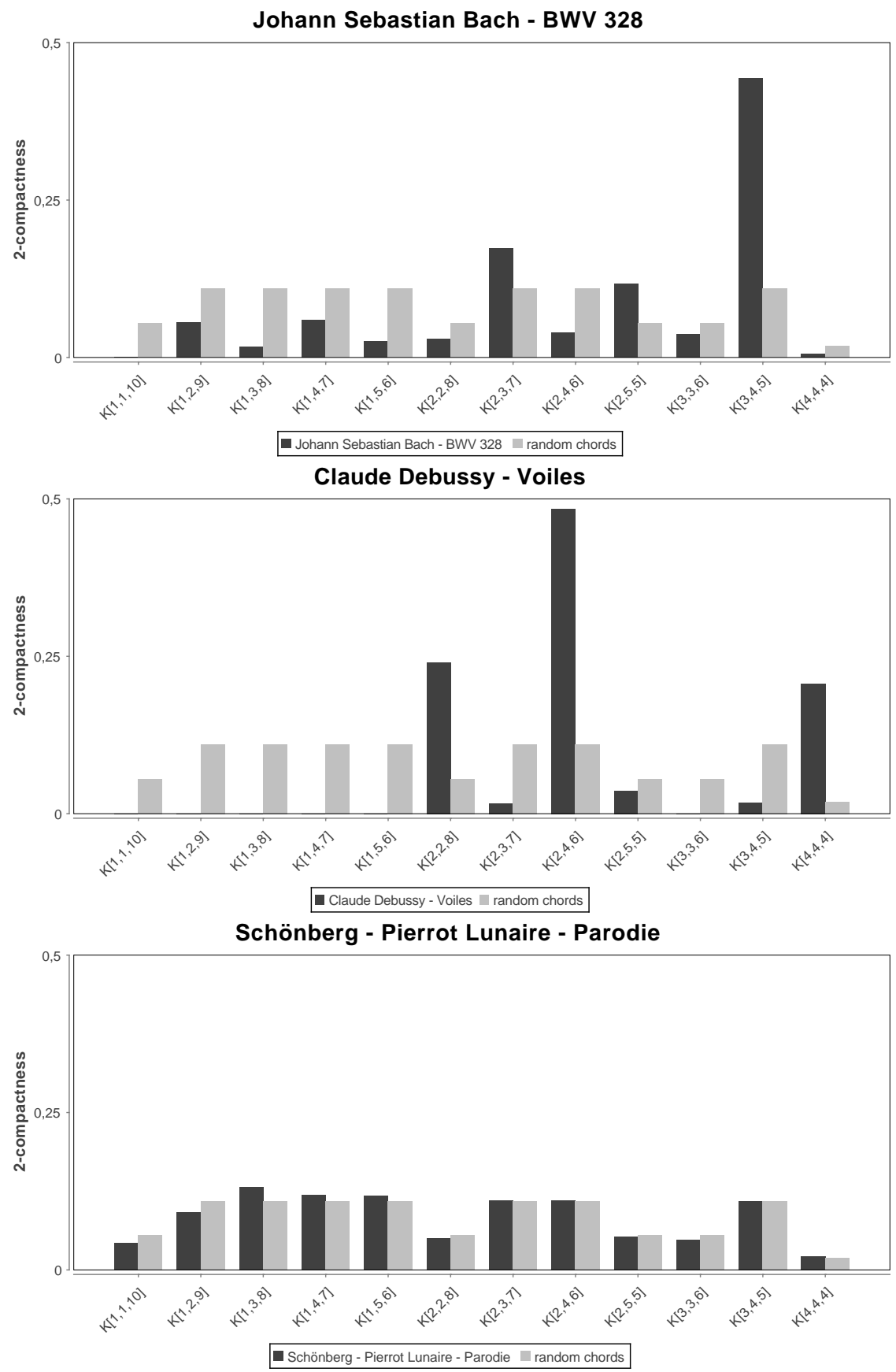

Fig. 3.17 2-compactness of the trajectories of three pieces in the 12 chromatic T/I complexes. In each complex, the compactness of the pieces (in black) is compared to the average compactness of chords (in grey) 
of pitch class sets of size 3 or more throughout the piece, as one might expect in twelve-tone or atonal music.

Three drastically different pieces have been chosen in this example in order to illustrate how the compactness of trajectories can depend on the musical style in an illuminating and informative way. However, this method can also be used to reveal more refined nuances between pieces in the same style.

\subsubsection{Distance and Classification}

The previous examples show that calculating the compactness of a piece in a set of complexes provides an abstract description that can highlight certain aspects of its harmonic structure. This description can be used to compare pieces as well. We propose a notion of distance between musical sequences based on compactness regarding a set of complexes. For a set of complexes $E=\left\{\mathcal{K}_{1}, \mathcal{K}_{2} \ldots\right\}$, we define the $d$-distance between two pieces $P$ and $P^{\prime}$ by the Euclidean distance:

$$
D_{E, d}\left(P, P^{\prime}\right)=\sqrt{\sum_{\mathcal{K} \in E}\left(\mathcal{C}_{d}\left(\mathcal{K}, T_{\mathcal{K}}\right)-\mathcal{C}_{d}\left(\mathcal{K}, T_{\mathcal{K}}^{\prime}\right)\right)^{2}},
$$

where $T_{\mathcal{K}}$ and $T_{\mathcal{K}}^{\prime}$ are the trajectories representing, respectively, $P$ and $P^{\prime}$ in $\mathcal{K}$. The $d$-distance is computed by only taking into account chords whose size is greater than or equal to $d+1$.

The mean compactness of the pieces constituting a corpus allows us to calculate a distance between any piece and this corpus. This notion of harmonic distance has been used for music classification (Bigo, 2013).

\subsubsection{Transformations on Trajectories}

The method described above allows any musical sequence to be represented by a trajectory within a given chord complex. Thanks to the symmetry properties of some T/I complexes, it is sometimes easy to have an immediate intuition of the musical interpretation of some geometrical transformations on trajectories or their embedding in different support spaces. In the first case we can, for example, interpret a transposition or an inversion operation as a translation or a rotation, respectively, of a given trajectory.

Figure 3.18 displays the trajectory representing the first measures of Bach's chorale, BWV 332, which is rotated by $180^{\circ}$. This operation transforms major chords into minor chords (and vice versa). A second type of transformation consists of the embedding of a given trajectory in a new support space. This corresponds to a relabelling of the notes attached to a trajectory according to the pitch content of the new underlying space. Although the trajectory remains the same, its embedding in a new space dramatically changes the intervallic relations between the notes. As an 

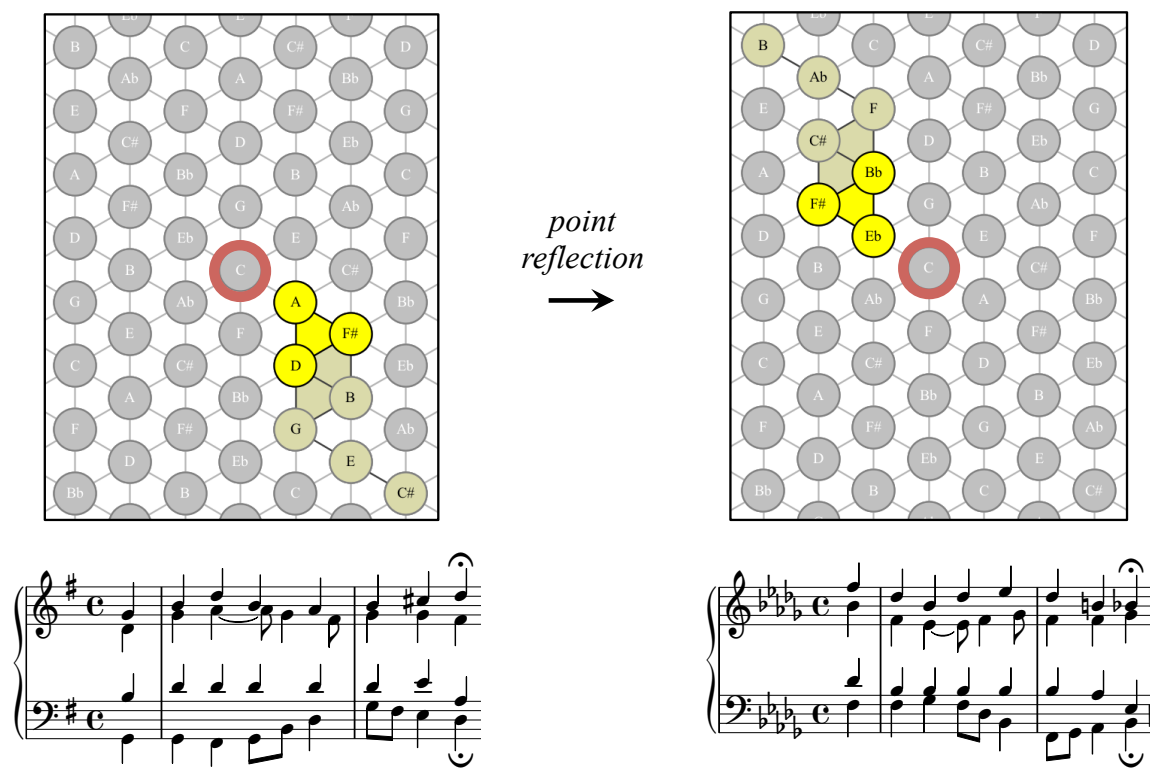

Fig. 3.18 On the left, a trajectory representing the first measures of the chorale, BWV 332, by J. S. Bach in $\mathcal{K}_{T I}[3,4,5]$. On the right, a point reflection is applied to this trajectory, producing a new sequence

example, Fig. 3.19 shows the result of embedding the trajectory in $\mathcal{K}_{T I}[3,4,5]$ of the first measures of the chorale, BWV 332, in the new support space, $\mathcal{K}_{T I}[2,3,7]$. This embedding produces a new musical sequence which will sound more exotic, due to the prominence of the pentatonic scale in the new support space. From a mathematical point of view, transformations of trajectories within chord complexes can be formalized as morphisms (Bigo et al., 2014).

\subsection{The HexaChord Software}

HexaChord is a freely available computer-aided music analysis software package based on the spatial representations presented in this chapter. ${ }^{6}$ The software provides a 3 -D visualization of the complex representing any arbitrary set of chords. To improve intelligibility, chromatic and diatonic T/I complexes of dimension 2 (i.e., constituted of 3-note chords) can be unfolded as infinite two-dimensional triangular tessellations, in the same style as the planar representation of the Tonnetz (see Figs. 3.2 and 3.12). The 12 chromatic T/I complexes together with the four diatonic T/I complexes, each

\footnotetext{
${ }^{6}$ http://www.lacl.fr/ lbigo/hexachord
} 

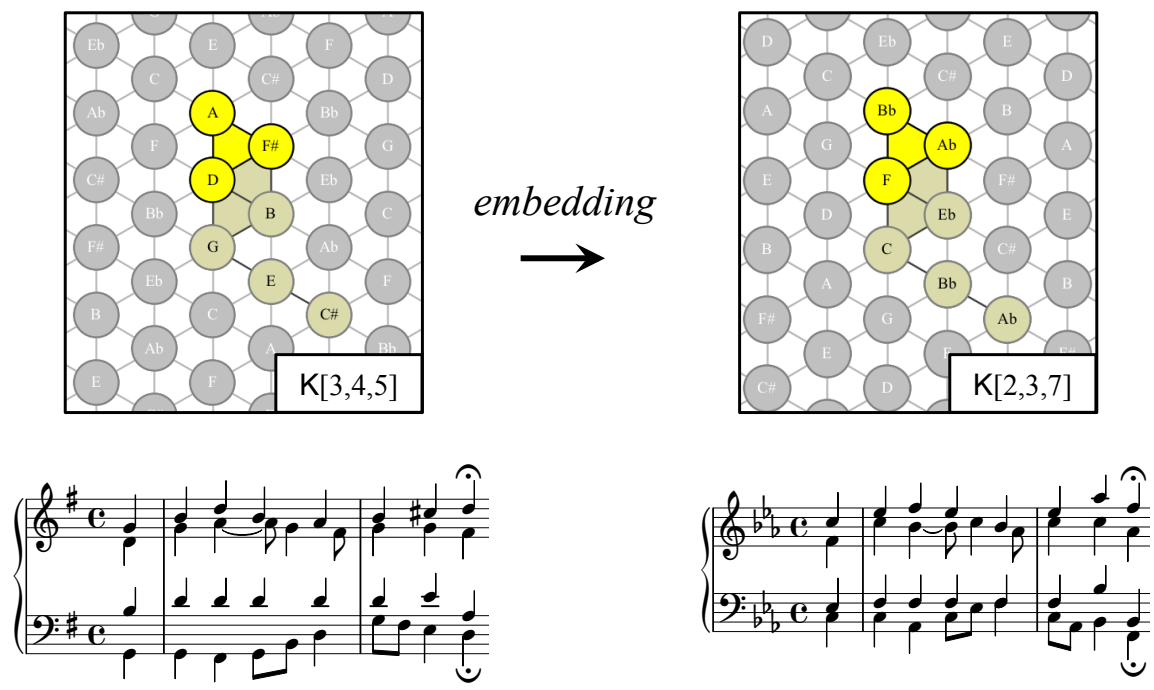

Fig. 3.19 On the left, the first measures of Bach's chorale, BWV 332, represented by a trajectory in $\mathcal{K}_{T I}[3,4,5]$. On the right, the transformation of the sequence resulting from the embedding of the trajectory in $\mathcal{K}_{T I}[2,3,7]$

available in the 12 diatonic scale, result in a total of 60 available planar organizations of pitch classes.

In the unfolded representation, pitch-classes and chords are repeated multiple times along interval axes in the complex. As a consequence, a piece can be represented by an infinite number of trajectories. Different strategies can then be applied to build a satisfying trajectory from a musical sequence (Bigo, 2013). An algorithm that minimizes the distance between every pair of successive chords in the complex will be more efficient for analysing chord progressions in time. On the other hand, an algorithm maximizing the compactness of each chord (independently of the other chords) will be more efficient for analysing the harmonic content of a piece.

Musical pieces are imported as MIDI files. A trajectory is automatically computed for any given piece-complex pair. The trajectory is represented as a path which evolves in real time within its complex while the piece is being played. The compactness of the trajectory over time (and on average) is automatically computed for any T/I complex and in any dimension. The calculation of compactness reveals the complexes which are the most harmonically related to the piece. Following this hypothesis, this functionality should suggest to the user which spaces can be used in order to reveal some interesting harmonic properties of a piece via its visualization.

HexaChord also allows the geometrical transformations described in Sect. 3.4.4 to be carried out on trajectories. A trajectory in a complex $\mathcal{K}$ can be translated, rotated, or even embedded in an other chord complex $\mathcal{K}^{\prime}$. Every spatial transformation causes the values labelling the sub-complexes constituting the trajectory to be refreshed. The harmonic and melodic content of the original piece can be transformed according 
to the new labels of the selected trajectory, thus leading to a new musical piece which can be exported in MIDI format or analysed and transformed using the spatial techniques described in this chapter.

\subsection{Conclusion}

In this chapter, we have presented a method for identifying any set of chords by a labelled simplicial complex. The family of T/I complexes relate to Generalized Tonnetze and can be used as support spaces to represent musical pieces for analysis and classification purposes.

The analysis method proposed here relates to the instantaneous verticality of chords. However, studying harmonic properties within a piece requires a horizontal approach as well (i.e., to study transitions between successive chords). In future work, we intend to address this issue by investigating methods for computing distances between sub-complexes within a complex.

We believe that the visual and intuitive aspects of this spatial approach constitute a strong pedagogical advantage for the understanding of harmony. For example, T/I complexes offer an original and intuitive reformulation of the notion of inversionally/transpositionally related chord classes. Moreover, we believe that the spatial reformulation of musical problems has a heuristic advantage. Thanks to its intuitive

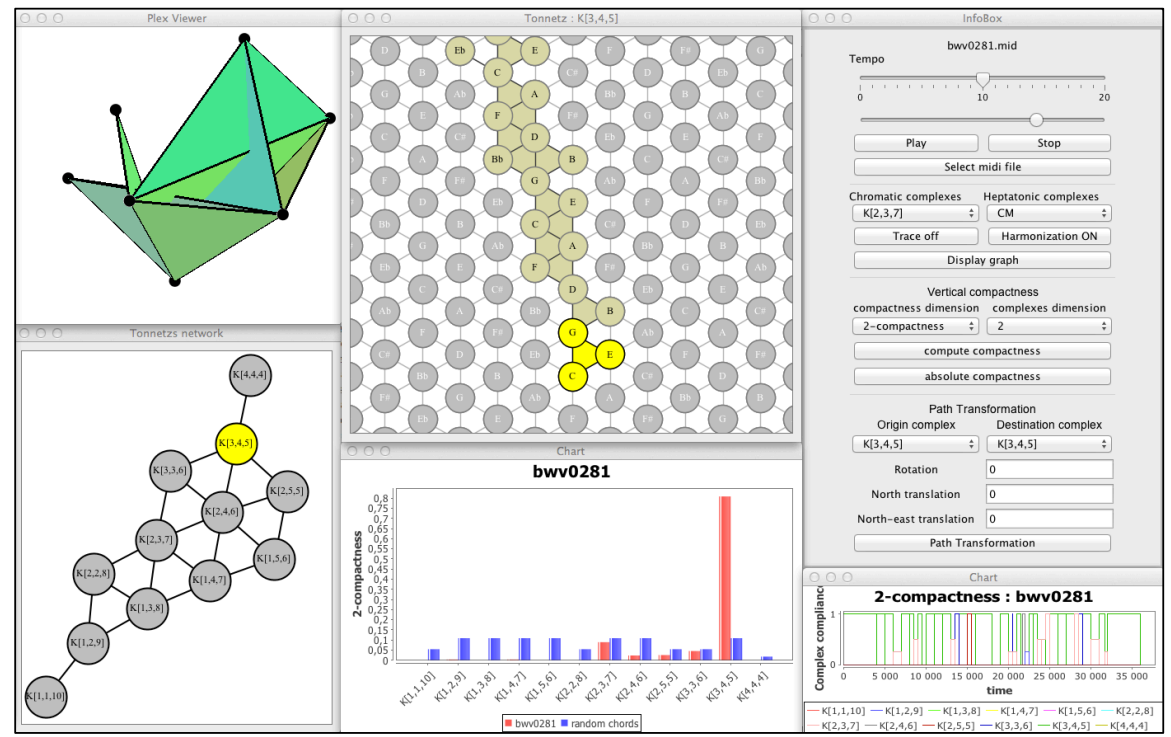

Fig. 3.20 Graphical user interface of HexaChord 
aspects, spatial structures frequently suggest unexpected questions, thus opening new research areas.

Finally, methods for automatically computing space-related descriptors (compactness of sub-complexes, distances within complexes, etc.) should allow for the construction of statistical models given a corpus of a particular style. Such models, based so far on viewpoints without spatial considerations, have been shown to be useful in various areas including music prediction and classification (Conklin and Witten, 1995). In future work we therefore intend to carry out an in-depth investigation of some statistical models built from spatial descriptions of musical pieces.

Acknowledgements The authors are very grateful to Antoine Spicher and Olivier Michel from LACL - Université Paris-Est, Jean-Louis Giavitto from IRCAM - Université Pierre et Marie Curie, Jean-Marc Chouvel from Université de Reims, and Darrell Conklin from Universidad del Pais Vasco for numerous fruitful discussions. This research is partially supported by the project "Learning to create" (Lrn2Cre8). The project Lrn2Cre8 acknowledges the financial support of the Future and Emerging Technologies (FET) programme within the Seventh Framework Programme for Research of the European Commission, under FET grant number 610859.

\section{References}

Andreatta, M. (2008). Calcul algébrique et calcul catégoriel en musique: Aspects théoriques et informatiques. In Pottier, L., editor, Le calcul de la musique, pages 429-477. Publications de l'Université de Saint-Etienne.

Andreatta, M. and Agon, C. (2003). Implementing algebraic methods in OpenMusic. In Proceedings of the International Computer Music Conference, Singapore.

Bigo, L. (2013). Représentations symboliques musicales et calcul spatial. PhD thesis, LACL/IRCAM, Université Paris-Est.

Bigo, L. and Andreatta, M. (2014). A geometrical model for the analysis of pop music. Sonus, 35(1):36-48.

Bigo, L., Ghisi, D., Spicher, A., and Andreatta, M. (2014). Spatial transformations in simplicial chord spaces. In Proceedings of the Fortieth International Computer Music Conference and Eleventh Sound and Music Computing Conference (ICMC/SMC 2014), pages 1112-1119, Athens, Greece.

Bigo, L., Giavitto, J.-L., and Spicher, A. (2011). Building topological spaces for musical objects. In Mathematics and Computation in Music: Third International Conference, MCM 2011, Paris, France, June 2011, Proceedings, volume 6726 of Lecture Notes in Artificial Intelligence, pages 13-28. Springer.

Callender, C., Quinn, I., and Tymoczko, D. (2008). Generalized voice-leading spaces. Science, 320(5874):346.

Catanzaro, M. (2011). Generalized Tonnetze. Journal of Mathematics and Music, $5(2): 117-139$.

Chew, E. (2002). The spiral array: An algorithm for determining key boundaries. In Music and Artificial Intelligence: Second International Conference, ICMAI 2002, Edinburgh, Scotland, UK, September 2002, Proceedings, pages 18-31. Springer. 
Chouvel, J.-M. (2009). Traversée du vent et de la lumière. Six remarques pour une phénoménologie de la création musicale. Available online at http://jeanmarc.chouvel.3.free.fr/textes/Traversee0.0.pdf.

Cohn, R. (1997). Neo-Riemannian operations, parsimonious trichords, and their "Tonnetz" representations. Journal of Music Theory, 41(1):1-66.

Cohn, R. (2012). Audacious Euphony: Chromatic Harmony and the Triad's Second Nature. Oxford University Press.

Conklin, D. and Witten, I. H. (1995). Multiple viewpoint systems for music prediction. Journal of New Music Research, 24(1):51-73.

Euler, L. (1739). Tentamen novae theoriae musicae ex certissismis harmoniae principiis dilucide expositae. Saint Petersburg Academy.

Forte, A. (1973). The Structure of Atonal Music. Yale University Press.

Giavitto, J.-L. and Michel, O. (2001). MGS: a rule-based programming language for complex objects and collections. In van den Brand, M. and Verma, R., editors, Electronic Notes in Theoretical Computer Science, volume 59, pages 286-304. Elsevier Science Publishers.

Giavitto, J.-L. and Spicher, A. (2008). Simulation of self-assembly processes using abstract reduction systems. In Krasnogor, N., Gustafson, S., Pelta, D., and Verdegay, J. L., editors, Systems Self-Assembly: Multidisciplinary Snapshots, pages 199-223. Elsevier.

Gollin, E. (1998). Some aspects of three-dimensional “Tonnetze”. Journal of Music Theory, 42(2):195-206.

Holland, P. W. and Leinhardt, S. (1971). Transitivity in structural models of small groups. Small Group Research, 2:107-124.

Hook, J. (2014). Generic sequences and the generic Tonnetz. Oxford Handbooks Online.

Mandereau, J., Ghisi, D., Amiot, E., Andreatta, M., and Agon, C. (2011). Z-relation and homometry in musical distributions. Journal of Mathematics and Music, $5(2): 83-98$.

Mazzola, G. et al. (2002). The Topos of Music: Geometric Logic of Concepts, Theory, and Performance. Birkhäuser.

Pousseur, H. (1968). L'apothéose de Rameau. Essai sur la question harmonique. Musiques Nouvelles. Revue d'esthétique, 21, pages 105-172.

Pousseur, H. (1998). Applications analytiques de la technique des réseaux. Revue belge de Musicologie, 52, pages 247-298.

Rameau, J.-P. (1722). Traité de l'harmonie, reduite à ses principes naturels. JeanBaptiste-Christophe Ballard.

Tymoczko, D. (2012). The generalized Tonnetz. Journal of Music Theory, 56(1):1-52. 